\title{
Experimental and numerical response of rigid slender blocks with geometri- cal defects under seismic excitation
}

\author{
Charlie Mathey ${ }^{1}$, Cyril Feau ${ }^{1}$, a , loannis Politopoulos ${ }^{1}$, David Clair ${ }^{2}$, Laurent Baillet ${ }^{3}$, and Michel Fogli ${ }^{2}$ \\ ${ }^{1}$ Laboratory of seismic mechanics \\ CEA/DEN/DANS/DM2S/SEMT/EMSI \\ 91191 Gif-sur-Yvette, France \\ ${ }^{2}$ Institut Pascal \\ UMR 6602 CNRS/UBP/IFMA \\ BP 80 026, 63171 Aubière, France \\ ${ }^{3}$ ISTerre, Université de Grenoble-Alpes \\ CNRS/IRD/IFSTTAR \\ BP 53, 38041 Grenoble, France
}

\begin{abstract}
The present work investigates on the influence of small geometrical defects on the behavior of slender rigid blocks. A comprehensive experimental campaign was carried out on one of the shake tables of CEA/Saclay in France. The tested model was a massive steel block with standard manufacturing quality. Release, free oscillations tests as well as shake table tests revealed a non-negligible out-of-plane motion even in the case of apparently plane initial conditions or excitations. This motion exhibits a highly reproducible part for a short duration that was used to calibrate a numerical geometrically asymmetrical model. The stability of this model when subjected to 2000 artificial seismic horizontal bidirectional signals was compared to the stability of a symmetrical one. This study showed that the geometrical imperfections slightly increase the rocking and overturning probabilities under bidirectional seismic excitations in a narrow range of peak ground acceleration.
\end{abstract}

\section{Introduction}

A free structure placed on the ground and not anchored is likely to rock or overturn facing an earthquake. The more the structure is slender, the more the risk of overturning is important. Thus, columns of ancient temples, gravestones or stacking containers are all kind of structures that may be concerned by this type of risk.

The numerical modeling of the rocking behavior of structures under seismic motion, is a quite challenging task since this problem includes several difficulties due to the large displacements and large rotations, and to the impact and friction nonlinearities which conduct to an extreme sensitivity of the responses of these structures to small perturbations [1].

One of the most frequently used models is Housner's model [2]. It considers a symmetric homogeneous rigid block in pure two-dimensional (2D) motion, assuming that impacts are instantaneous and non-elastic, occuring only at the edges of the block with sticking contact conditions. Since, a lot of authors have improved this model, relaxing some of the assumptions but dealing mainly with ideal

\footnotetext{
ae-mail: cyril.feau@cea.fr
}

blocks (i.e. prismatic, homogeneous, symmetric and perfect contact, e.g. [3]).

The present work focuses on the influence of small geometric defects on the behavior of slender rigid blocks, to investigate of their stability under seismic excitation. To this end, an experimental and analytical study of the response of non-perfect slender blocks was undertaken. A comprehensive experimental campaign was carried out on one of the shake tables of CEA/Saclay in France (section 2). The tested model was a massive steel block with common manufacturing quality.

The rigid block motion measurement is usually done by chrono-photography or recently by videometry (e.g. [4]). The originality of our campaign is that we used rotational velocity sensors. Indeed, the high accuracy of these sensors enabled us to highlight an out-of-plane (3D) motion even in the case of apparently plane initial conditions or excitations. This behavior exhibiting a highly reproducible part for a short duration, this was used to calibrate a numerical geometrically asymmetrical model (section 3).

Then, the stability of a rigid block with geometrical defect is numerically compared to the stability of its al- 


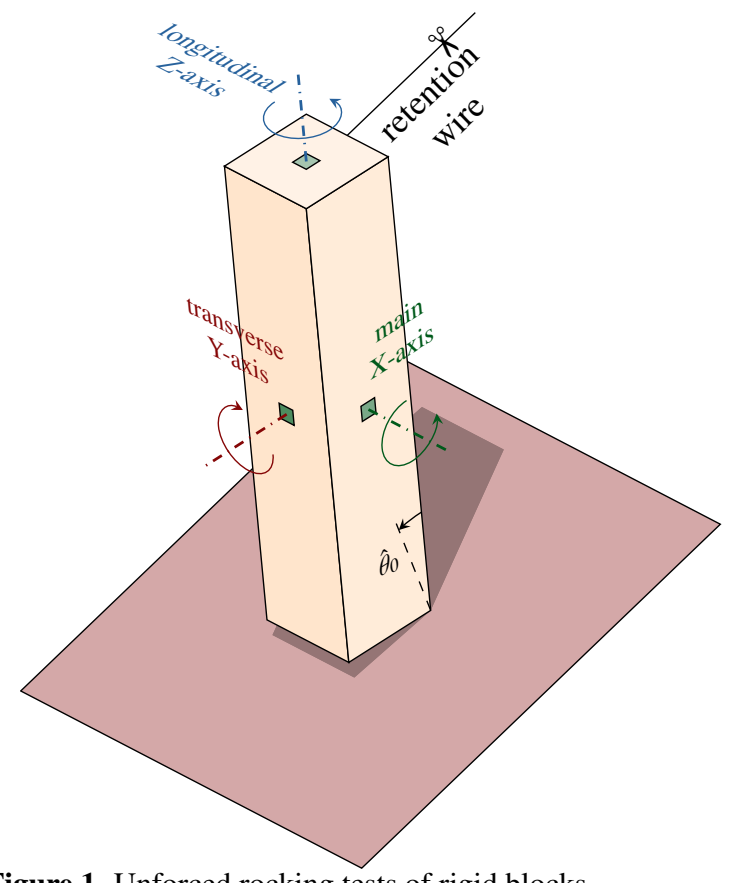

Figure 1. Unforced rocking tests of rigid blocks

ter ego with perfect geometry by submitting them to 2000 pairs of artificial seismic horizontal signals.

\section{Experimental campaign}

\subsection{Experimental set up}

In order to gain further insight into the dynamic response of rigid blocks and to validate a numerical model, an experimental campaign limited to release tests was carried out (see Figure 1).

They provide useful information on the details of the dynamic response, including 3D effects, and thus are well suited to validate analytical models. To impose the initial conditions during the release tests, (i) the block was tilted by pulling its top with a steel wire. The imposed tilt angle $\hat{\theta}_{0}$ was measured with a displacement sensor and (ii) the wire was cut releasing the block, letting it free to oscillate on a rigid floor.

A massive steel block with a slenderness of $7(10 \mathrm{~cm} \times$ $10 \mathrm{~cm} \times 70 \mathrm{~cm}$ ), of about $54 \mathrm{~kg}$ has been manufactured to be used for the tests. This high slenderness has been chosen to avoid sliding, in accordance with Housner's classical model assumptions [2]. Four feet have been machined monolithically with the block to obtain a specimen with as little uncertainties as possible with respect to its design.

To measure the 3D motion of the block, the block has been instrumented with three angular velocity sensors (range: $\pm 200^{\circ} \mathrm{s}^{-1}$, accuracy: $\pm 0.01^{\circ} \mathrm{s}^{-1}$ ) mounted on the three faces of the block (green patches in Figure 1). These sensors measure the angular velocities in the convected frame which follows the rotation of the block.

Therefore, the comparison between analytical and experimental results will be made on the basis of these quantities, called convected rotational velocities (CRV). The displacement measurements were not considered (i) thanks to a suitable choice of the slenderness of the body (ii) because this type of measurement is quite challenging without affect the response of an unrestrained rocking body. Nevertheless, the possible sliding and bouncing behaviors have been taking into account in the numerical simulations.

\subsection{Experimental results}

This section discusses, on a phenomenological basis, the experimental results shown in Figure 2. For the sake of readability, only the results of eight tests are presented. However, it must be noticed that the observed characteristics of the response are confirmed by a series of other similar tests.

Despite a lot of care to obtain pure 2D motion, in addition to rotation velocity around the main axis, nonnegligible rotation velocities around the transverse and longitudinal axes are also measured. The maximum CRV around these two axes is about $10 \%$ of the one around the main axis. In the authors' opinion, this unexpected out-ofplane motion must not be neglected a priori. Hence the underlying origin of this 3D response must be identified and taken into account, especially for the study of the stability of such structures under seismic excitation.

To describe the experimental results, the notion of main impact (MI) is defined as an impact which corresponds to a local maximum of the time history of the CRV around the main $\mathrm{X}$-axis (Figure $2 \mathrm{a}$ ). An out-of-plane motion occurs with the first MI. Between the first and the second MIs the motion follow a quite complex but reproducible pattern. The following observations can be made:

- The CRV around the transverse axis (Figure 2c) has a quasi-constant, slightly convex envelope, and varies linearly between positive and negative values.

- The CRV around the longitudinal axis (Figure 2d), after a first peak quasi-concomitant with the main impact, exhibits a highly concave envelope which is almost equal to zero at the beginning and at the end of the interval. The CRV varies from positive to negative values, following a curve composed of two segments.

- The CRV measured around the main axis is not smooth. A slight "sawtooth" pattern is superposed to the main curve. This pattern is synchronous with the transversal and longitudinal oscillations (Figure $2 b$ ).

Given that all runs show a quasi-identical 3D motion until the second MI, the numerical model should predict, with high accuracy, the CRV during this time interval. Moreover, the above repeatable response suggests that the initiation of the out-of-plane motion has a deterministic cause, which is the same for all runs. Since the block was not placed exactly on the same spot before each test, the authors assume that the 3D behavior is induced by an asymmetry of the block itself and not by local defects of its support plate. 


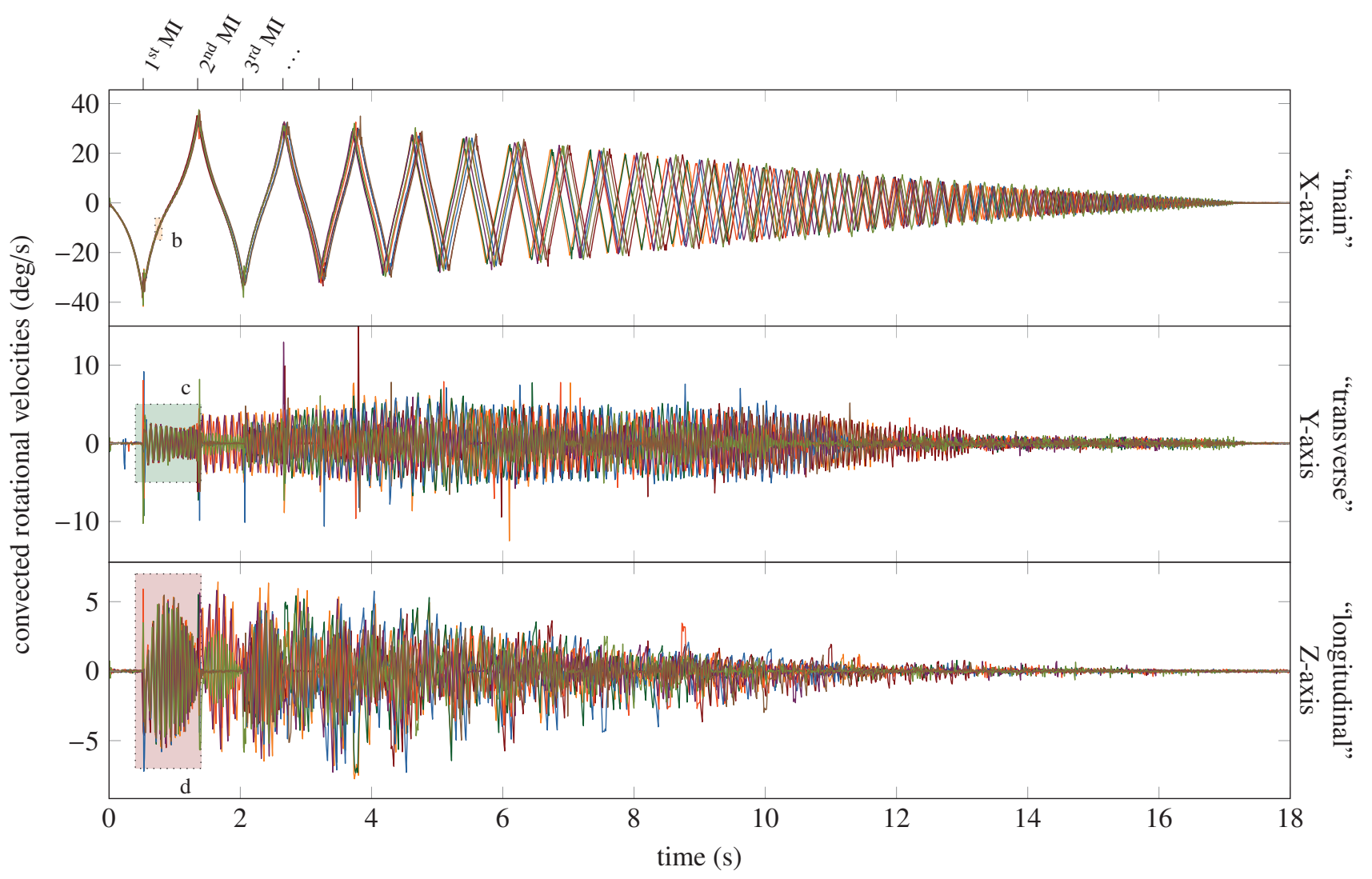

(a)

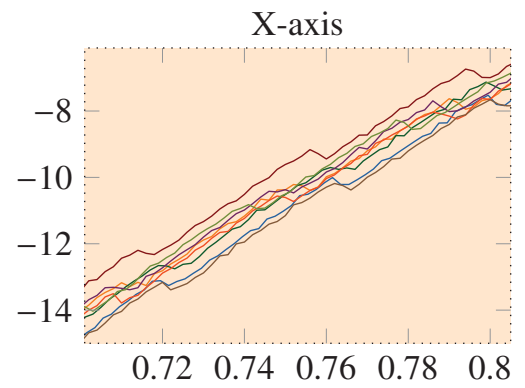

(b)

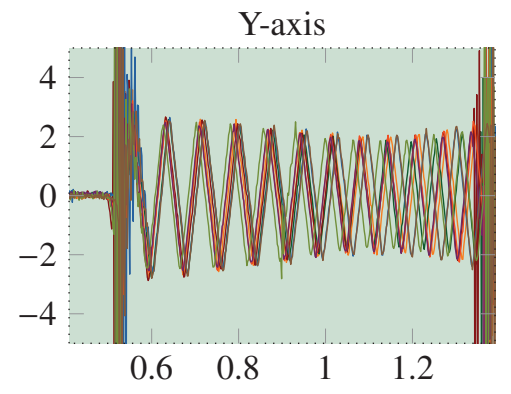

(c)

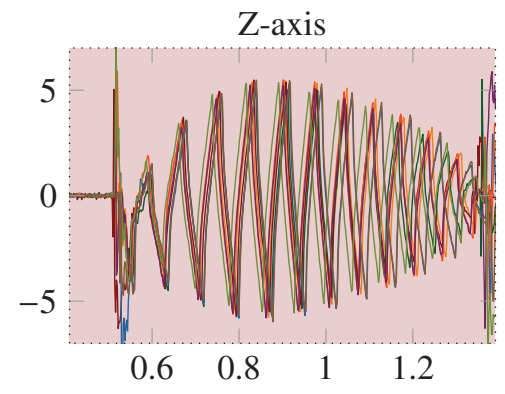

(d)

Figure 2. Experimental results of eight runs. For all figures, abscissae are the time (s) and ordinates are the convected rotational velocities $(\mathrm{deg} / \mathrm{s})$ around the specified axis.

2a: Global results of the 8 runs.

2b to 2d: Zoom in on Figure 2a focusing on the 3D pattern used to calibrate the numerical model.

\section{Numerical model}

This section presents the basic assumptions of the numerical model which has been developed and the method that has been used to determine the values of its parameters. Eventually, a comparison between the model and the experimental results is presented.

\subsection{Numerical assumptions}

To carry out comprehensive series of analyses such as Monte Carlo simulations, a simple and costly effective model should be used. Therefore the block is assumed to be infinitely rigid and its motion can be described by only three translation and three rotation variables. To avoid the gimbal-lock problem, Simo's algorithm [5] deals with large rotations using unit quaternions (also known as versors). Moreover, it uses the conservation of momentum instead of Newton's law of motion as its main equation. Thus, the algorithm maintains the total momentum constant, unconditionally, for any time-step and despite the large rotations; an ability not present in Newmark's explicit algorithms. Finally, the natural outputs of Simo's algorithm are convected rotational velocities, which are also the quantities measured experimentally. 


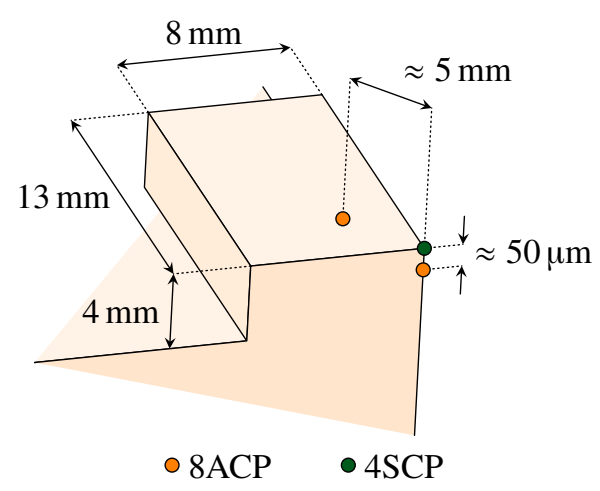

Figure 3. Geometry of a foot of the block and positions of the modeling nodes.

The four feet of the block are not modeled explicitly but are represented as nodes. Impact and friction are modeled by the penalty method [6]. Nevertheless, in this case, penalty parameters are not purely numerical artifices used to impose contact conditions. Instead, they reflect physical, local flexibility and dissipation of the feet that are not taken into account by the rigid body model of the block. In the present case, the problem of contact is quite complex since contact points can both impact the plate support instantaneously with a moderate velocity (between 0.01 and $10 \mathrm{~m} \mathrm{~s}^{-1}$ ) and remain in contact for some time.

In this work, a modified Kelvin-Voigt's law is used for the normal contact force component, and the Coulomb's law for tangential component (more details can be found in [7]).

The out-of-plane motion can be explained only by introducing some defects in the model. At the early stages of this study, several possible defects were considered, for instance, asymmetrical impact stiffness or unequal feet lengths. In this latter case, initially, only four contact points (i.e. one contact point per foot) were considered. Nevertheless some features of the motions could not be accurately reproduced. That is why, the numerical model was enriched by introducing four supplemental contact points (one supplemental contact point per foot, see Figure 3). This eight asymmetric contact points (8ACP) model is an effort to account, in a simplified fashion, for inevitable defects at the individual foot level, due to its finite size.

\subsection{Parameter identification}

The geometrical defects of the 8ACP model have to be large enough to cause a non-negligible out-of-plane motion and have to be too small to be measured by usual instruments. Moreover, the values of the penalty parameters (normal and tangential stiffness and damping for each contact point) are not known a priori. Therefore the values of these parameters have been determined through an optimization procedure by means of an evolutionary algorithm.

This algorithm consists in minimizing an error or cost function which is a measure of the divergence between the results given by a numerical model, itself defined by a set of parameters called "chromosome", and a reference. In the present case, the numerical model is the above model of the block with defects and the reference is the experimentally recorded response of a release test. At each iteration, called "generation", the best chromosomes are used to create the new chromosomes that have to be tested.

The definition of the cost function is essential to obtain relevant results. This is not an easy task and should be carried out empirically guided by the specificity of the physical processes and engineering judgment. In the present case, the evolutionary algorithm focuses on the reproducible out-of-plane motion presented on Figures 2c and $2 \mathrm{~d}$. The error function is not presented here because it cannot be expressed in a simple closed form.

\subsection{Numerical results}

Figure 4 shows the experimental and numerical CRV. In addition to the CRV, the contact time-histories for each point of the 8ACP model are shown (the line is drawn as long as the block remains in contact).

The analytical model should be able to reproduce accurately the response between the first and the second main impacts which has been quasi-identical for all runs. The proposed analytical model meets this requirement. In fact the transversal and longitudinal motions are well reproduced (Figure $4 \mathrm{c}$ and $4 \mathrm{~d}$ ). The numerical and experimental motions follow the same sawtooth like pattern (Figure 4b), share the same slightly convex envelope and have the same frequency and phase.

After the second MI, the experimental and numerical time-histories may diverge more. Nevertheless, this divergence is within the range of variability of the results which corresponds to the various runs of the experimental campaign (Figure 2). In particular, the instant of transition between the 3D behavior and the quasi-2D behavior which occurs approximatively at time $t=10 \mathrm{~s}$ is also well reproduced.

Since, the block rocking motion is very sensible to small variations in the experimental or numerical conditions, an empirical sensibility analysis has been carried out to have an insight into the sensibility of the numerical model considering small variations on the model's parameters (e.g. time-step of numerical calculation, values of the mechanical and geometrical parameters and the initial condition $\hat{\theta}_{0}$ ). Though not shown here for the sake of conciseness, this analysis shown that the induced variability on the results is in the range of the experimental variability.

\section{Influence of the defects on the stability of a rigid block under seismic excitation}

To gain a better insight into the influence of geometrical defects on the stability of a slender rigid block submitted to seismic excitations, a statistical numerical study was carried out. In addition to the asymmetrical model (8ACP) presented in section 3 , a symmetrical model with only four 


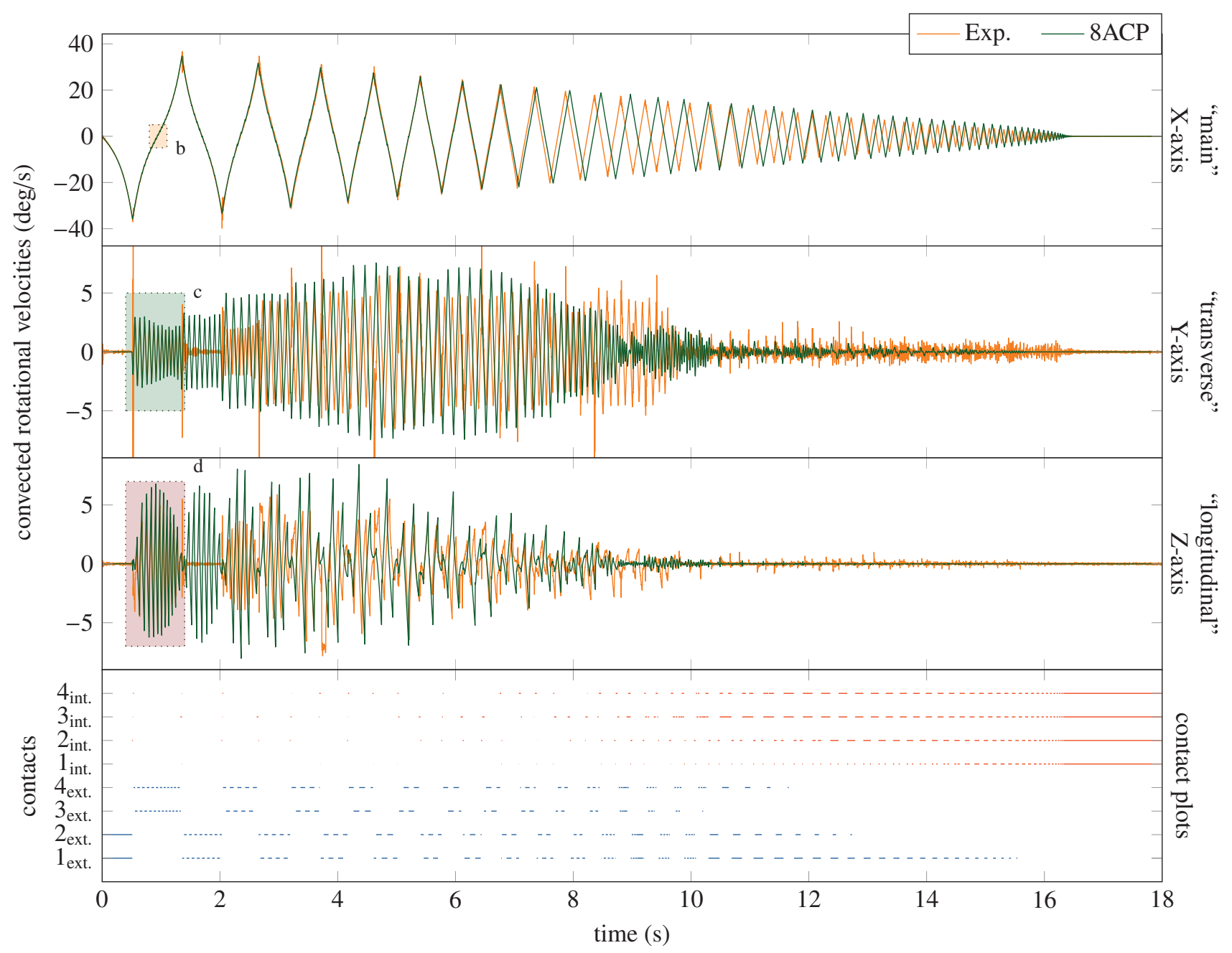

(a)

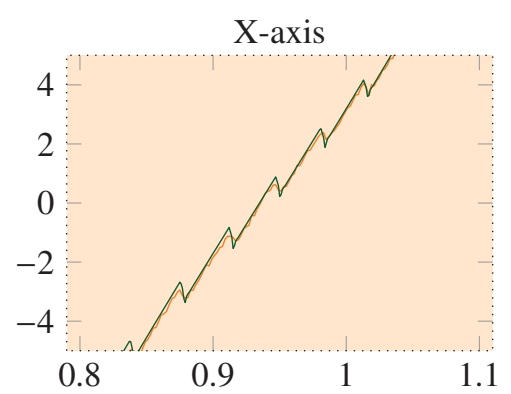

(b)

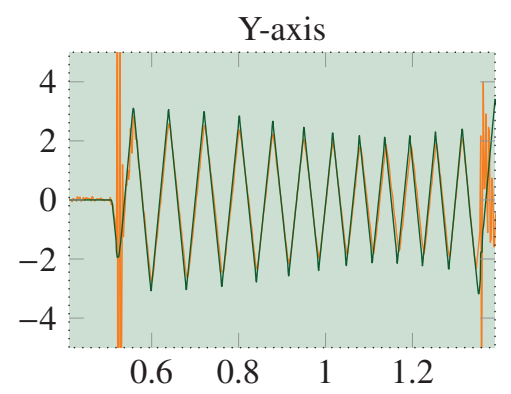

(c)

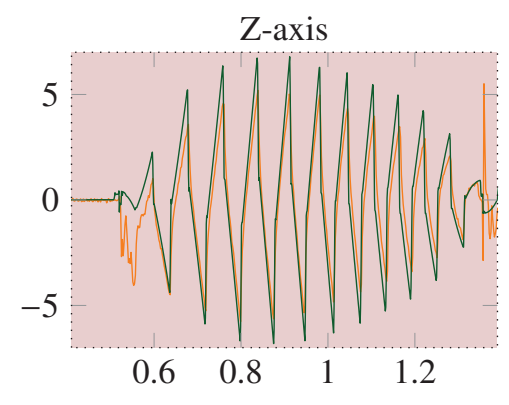

(d)

Figure 4. Comparison between experimental and numerical results. For all figures, abscissae are the time (s) and ordinates are the convected rotational velocities (deg/s) around the specified axis.

4a: Global comparison and contact plots.

4b to 4d: Zoom in on Figure 4a focusing on the 3D pattern.

exterior contact points (4SCP, see Figure 3), more likely to be used by practitioners, was considered. The values of the parameters of this model (stiffnesses, damping ratios and friction coefficient) correspond to the values of the four exterior points of the $8 \mathrm{ACP}$ model.

\subsection{Comparison protocol}

Several simulations were carried out using different artificial signals obtained by simulating realizations of a zeromean stationary Gaussian process then multiplying each simulated realization by a given function of time (called modulation, or envelope function). The power spectral density (PSD) used for the underlying stationary Gaussian 
process is the Kanaï-Tajimi spectral model [8] in which the frequency and the damping ratio were taken equal to $2.95 \mathrm{~Hz}$ and $55 \%$ respectively. These values lead to a PSD quite close to the PSD advocated by the Eurocode 8 for medium soil conditions [9]. This process was simulated using an algorithm based on the spectral representation of second-order stationary random processes [10]. The used modulation function is of the form $t^{u} \times \mathrm{e}^{-v t}$, where $u$ and $v$ were selected so that the total duration $T$ is about $10 \mathrm{~s}$ and the duration of maximum amplitude oscillations is about $3.5 \mathrm{~s}(u=2$ and $v \approx 1.26)$.

2000 pairs of accelerograms in the two horizontal directions, $\gamma_{\mathrm{gr} .}^{\mathrm{N}-\mathrm{S}}(t)$ and $\gamma_{\mathrm{gr} .}^{\mathrm{E}-\mathrm{W}}(t)$, were generated and scaled so that their peak values varied from $0.01 \mathrm{~g}$ to $0.5 \mathrm{~g}$.

Figure 5 shows a sample of the bidirectional excitation signals and the numerical results which correspond to the asymmetrical (8ACP) and symmetrical (4SCP) models. This figure illustrates the divergence of the earthquake responses of a rigid block, due to different modelling assumptions. Of course, in the case of a bidirectional excitation, the notions of main and transversal axes do not make sense, that is why they are only noted $\mathrm{X}$ and $\mathrm{Y}$ in the figure.

To obtain a statistical estimate of how these defects influence the response of a rigid block under seismic excitations, we considered as a relevant index of rocking amplitude and even overturning, the maximum of the vertical displacement of the external contact points $\mathcal{H}$ :

$$
\mathcal{H}=\max _{t \in[0, T]}\left(\max _{i \in 1 \cdots 4}\left(H_{i_{\text {ext. }}}(t)\right)\right)
$$

where $H_{i_{\text {ext. }}}(t)$ is the vertical displacement of the exterior point $i$ at time $t$.

\subsection{Statistical results}

The results of the empirical statistical study are shown in Figure 6, which represents the scatter plot of the maximum vertical displacement $\mathcal{H}$ versus the peak ground acceleration (PGA) of the two-directional excitation.

$$
\mathrm{PGA}=\max _{t \in[0, T]}\left(\sqrt{\left(\gamma_{\mathrm{gr} .}^{\mathrm{N}-\mathrm{S}}(t)\right)^{2}+\left(\gamma_{\mathrm{gr} .}^{\mathrm{E}-\mathrm{W}}(t)\right)^{2}}\right)
$$

For the sake of better readability, only 350 randomly chosen points are displayed in the interval $[0.1 \mathrm{~g}, 0.3 \mathrm{~g}]$. Moreover, the intermediate portion of the vertical axis is not shown because of the considerable range of possible values of the maximum vertical displacement. In fact, when a block has overturned, the maximum of the vertical displacement of the contact point exceeds the threshold $\mathcal{H}=10 \mathrm{~cm}$. The equilibrium point for a block resting on two feet is given for indicative purpose: $\mathcal{H}=$ $10 \mathrm{~cm} \times \sin (\arctan (1 / 7)) \approx 1.41 \mathrm{~cm}($ drawn with a dashed line).

It may be observed that the responses of the two models are different, especially for the class of PGA displayed by a colored background. This class $(0.170-0.194 \mathrm{~g})$ corresponds to weak excitation levels, yet higher than the theoretical PGA which induces rocking motion [2, 11]:

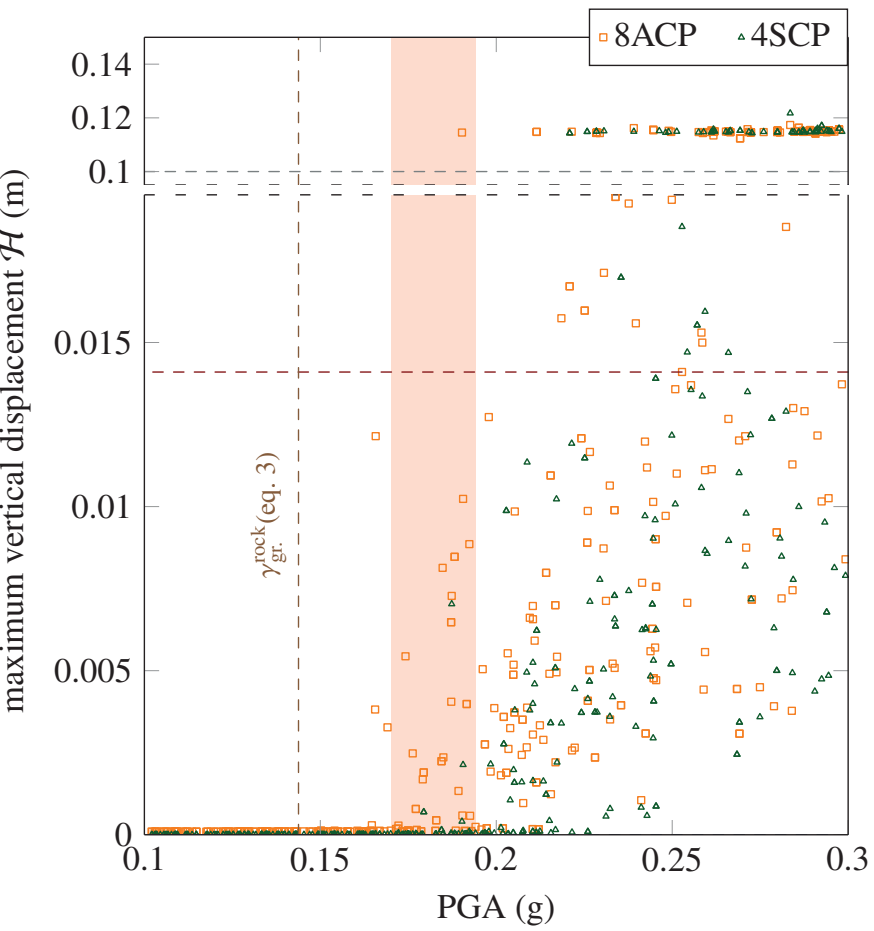

Figure 6. Evolution of the maximum vertical displacement according to the PGA

$$
\gamma_{\mathrm{gr} .}^{\text {rock }}=g \times \arctan (1 / 7) \approx 0.144 \mathrm{~g}
$$

Thus, in this class, bigger values of vertical displacements of contact points are obtained and even the overturning in the case of the asymmetrical model, contrary to the case of the symmetrical model.

\section{Conclusion}

In this work the experimental and analytical response of a slender rigid block in release conditions have been investigated. Several experimental tests have revealed a 3D behavior. A special care has been given in the description of the quasi-identical 3D behavior exhibited during the tests. Then, a numerical model of an asymmetrical rigid block with geometrical defects has been presented. In this model, eight contact points have been considered, in order to account, as simply as possible, for inevitable defects at the individual foot level, due to its finite size. In this work, the overall parameters of the numerical model have been determined using an evolutionary algorithm. Finally, to have a better insight into the influence of geometrical defects on the stability of a slender rigid block submitted to seismic excitations, a statistical numerical study has been performed considering the asymmetrical model and a symmetrical one (more likely to be used by practitioners). Thus, 2000 responses of each model have been calculated considering bidirectional synthetic excitations scaled with peak ground accelerations varying from $0.1 \mathrm{~g}$ to $0.5 \mathrm{~g}$. It is found that the model with defects is more prone to rocking and overturning than the model without defects. In particular, the two models lead to significantly different responses in the case of excitation levels slightly higher than 


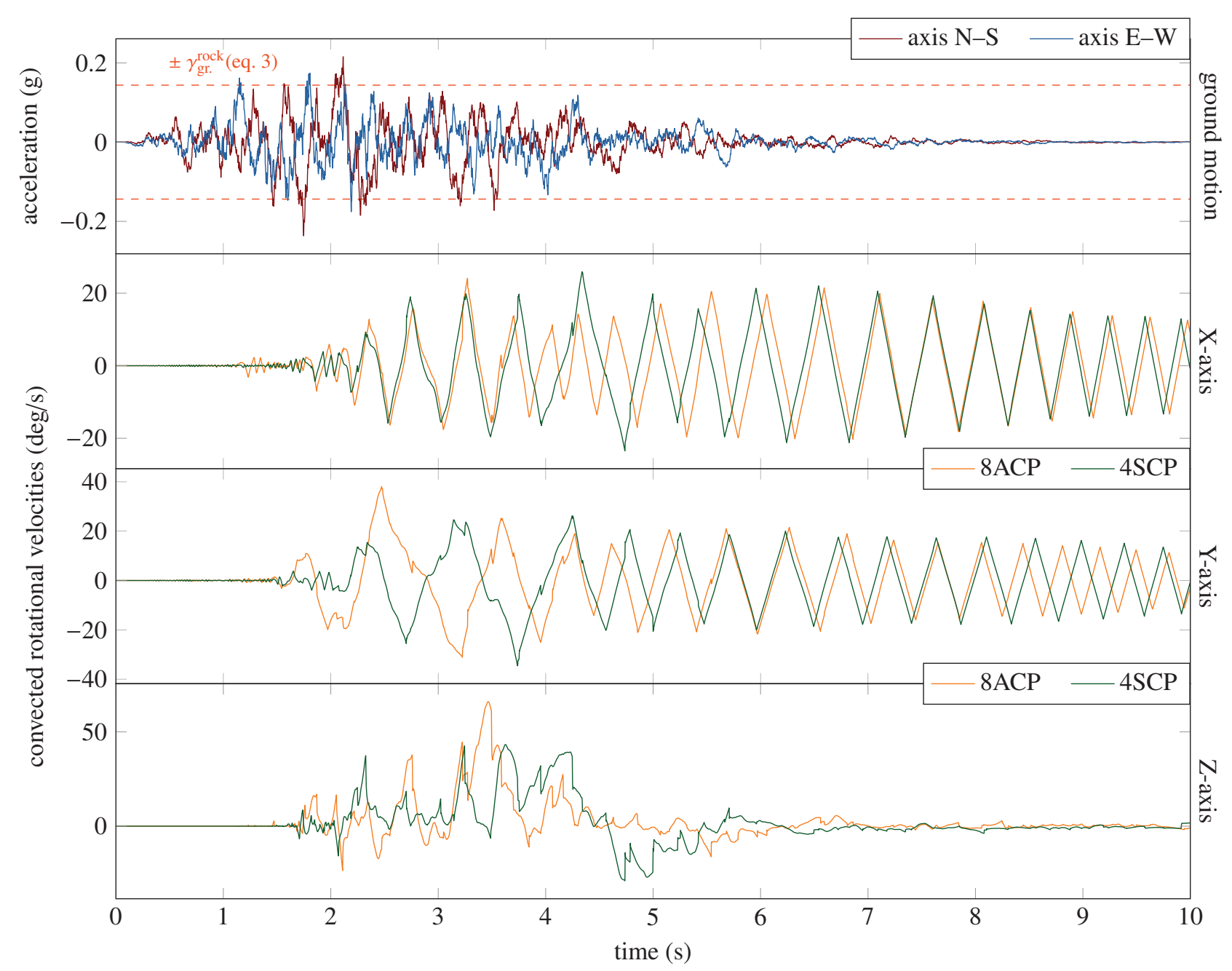

Figure 5. A comparison between $4 \mathrm{SCP}$ and $8 \mathrm{ACP}$ models

the theoretical PGA which induces rocking motion. The validity of the proposed analytical model was further investigated by its comparison with a series of seismic tests of similar slender blocks, carried out on the shake tables of CEA/Saclay in France.

\section{References}

[1] M. Jeong, K. Suzuki, S. Yim, Journal of sound and vibration 262, 1091 (2003)

[2] G. Housner, Bulletin of the Seismological Society of America 53, 403 (1963)

[3] I.N. Psycharis, P.C. Jennings, Earthquake Engineering \& Structural Dynamics 11, 57 (1983)

[4] P. Lipscombe, S. Pellegrino, Journal of engineering mechanics 119, 1387 (1993)

[5] J. Simo, N. Tarnow, Zeitschrift für angewandte Mathematik und Physik ZAMP 43, 757 (1992)
[6] J. Oden, J. Martins, Computer methods in applied mechanics and engineering 52, 527 (1985)

[7] C. Mathey, Tech. Rep. DEN/DANS/DM2S/SEMT/EMSI/RT/14-039/A,

Commissariat à l'énergie atomique et aux énergies alternatives (2015)

[8] Y. Lin, Y. Yong, Journal of engineering mechanics 113, 1119 (1987)

[9] P. Pinto, I. Vanzi, Base isolation: reliability for different design criteria (Tenth world conference on earthquake engineering, 1992)

[10] S.M. Prigarin, Spectral models of random fields in Monte Carlo methods (VSP, 2001)

[11] Y. Ishiyama, Y. Matsushima, Y. Yamazaki, S. Nakata, Vibration Test and Theoretical Consideration on Overturning of Furniture During Earthquakes, in Report of the Annual Meeting (1978), pp. 834-844 